This item was submitted to Loughborough's Research Repository by the author.

Items in Figshare are protected by copyright, with all rights reserved, unless otherwise indicated.

\title{
Introduction to the issue on signal processing for exploiting interference toward energy efficient and secure wireless communications
}

PLEASE CITE THE PUBLISHED VERSION

http://dx.doi.org/10.1109/JSTSP.2016.2620703

PUBLISHER

(C) IEEE

VERSION

AM (Accepted Manuscript)

LICENCE

CC BY-NC-ND 4.0

\section{REPOSITORY RECORD}

Krikidis, loannis, Christos Masouros, Gan Zheng, Rui Zhang, and Robert Schober. 2019. "Introduction to the Issue on Signal Processing for Exploiting Interference Toward Energy Efficient and Secure Wireless Communications". figshare. https://hdl.handle.net/2134/23627. 


\title{
Signal Processing for Exploiting Interference towards Energy Efficient and Secure Wireless Communications
}

\author{
Ioannis Krikidis, Senior Member, IEEE, Christos Masouros, Senior Member, IEEE, Gan Zheng, Senior Member, \\ IEEE, Rui Zheng, Senior Member, IEEE, and Robert Schober, Fellow, IEEE
}

I NTERFERENCE has been the central focus for meeting the ever increasing requirements on quality of service in modern and future wireless communication systems, and has long been considered as a deleterious factor that limits the system capacity. In conventional communications systems, the design objective is to allow users to share a medium with minimum or no interference. Thus, great efforts are made to avoid, mitigate, and cancel interference. For instance, to support multiple users, orthogonal access methods in time, frequency, code as well as spatial domains have been used in different generations of cellular systems. In future-generation heterogeneous cellular networks, due to the increasing number of uncoordinated low-power nodes such as femtocells to improve the coverage and capacity, interferences need to be mitigated in multiple domains, rendering their management a challenge. Interference mitigation/avoidance techniques provide convenient mechanisms to allow multiple users to share the wireless medium. However, they lead to inefficient use of wireless resources.

Contrary to this traditional view, which treats interference as a detrimental phenomenon, recent interest has emerged on innovative approaches that consider interference as a useful resource for developing energy efficient and secure $5 \mathrm{G}$ communication systems. These include exploiting constructive interference as a source of useful signal power at the modulation level by use of practical multiuser downlink precoding, and also the use of radio frequency radiation for energy harvesting that handles interference and unintended signals as a source of green energy. These techniques open new exciting opportunities in wireless communications by enabling energy self-sustainable and environmentally friendly networks with extended lifetimes, untethered mobility and independence from the power grid, and joint distribution of information and energy within networks. Further innovative uses of interference

I. Krikidis is with the Department of Electrical and Computer Engineering, University of Cyprus, Cyprus. E-mail: krikidis@ucy.ac.cy.

C. Masouros is with the Department of Electronic and Electrical Engineering, University College London, UK. E-mail: c.masouros@ucl.ac.uk.

G. Zheng is with the Wolfson School of Mechanical, Electrical and Manufacturing Engineering, Loughborough University, Loughborough, U.K. E-mail: g.zheng@lboro.ac.uk.

R. Zhang is with the Department of Electrical and Computer Engineering, National University of Singapore, Singapore. E-mail: elezhang@nus.edu.sg.

R. Schober is with the Institute for Digital Communications, FriedrichAlexander University Erlangen-Nurnberg, Erlangen, Germany. E-mail: rschober@ece.ubc.ca. include exploiting it for physical (PHY) layer secrecy, as an efficient means to jam potential eavesdroppers. This is particularly useful in networks without infrastructure to secure wireless links without the computational overhead imposed by standard cryptographic techniques. These research streams introduce a new vision about interference in wireless networks and motivate a plethora of potential new applications and services. The purpose of this special issue is to re-examine the notion of interference in communications networks and introduce a new paradigm that considers interference as a useful resource.

The above topics are still widely open individual research areas. Interference exploitation mainly focuses on precoding for conventional single-cell downlinks, while further more comprehensive studies are required in multicell scenarios; in the presence of channel state information errors, fundamental communication theoretic studies on the benefits of interference exploitation are needed; and application in the candidate 5G technologies such as massive multiple-input multiple-output (MIMO) and millimeter wave communications are of high importance. While there have been considerable efforts on energy harvesting $(\mathrm{EH})$ in the information and communication theoretical literature, open problems include the network level optimization of $\mathrm{EH}$, enabling techniques in the form of joint optimization of baseband and radio-frequency processing for $\mathrm{EH}$, the impact of received waveforms on the information and power transfer efficiency amongst others. Similarly, open problems exist in PHY layer secrecy research, examples include the design of corresponding enabling techniques for $5 \mathrm{G}$ scenarios and candidate technologies, the impact of non-Gaussianity of the interference on PHY layer secrecy, and PHY layer secrecy in full-duplex networks with self-interference.

More importantly, while the above are still individually open research areas, future practical communication systems will require the co-existence of the above technologies. The enabling techniques, optimization designs, and theoretical studies for a) providing secrecy in an energy erative environment, b) ensuring security with modulation-level interference exploitation systems, c) combining EH together with constructive interference to enhance both the received signal quality and the energy levels of the receivers are still unexplored research realms. Therefore, the joint optimization, joint information theoretic studies, and joint enabling signal processing techniques combining the above are widely open research topics and form the central motivation behind this special issue. 
We received a total of 68 submissions, spanning a broad range of topics from PHY layer secrecy, cooperative networks, large antenna systems, wireless power transfer, cognitive radio, and interference management. After a thorough peer-review process, 16 articles have been selected and are presented in the following discussion.

The first twelve papers deal with the exploitation of interference as an efficient way to increase PHY layer secrecy. In the first article, Zhao et. al. propose a PHY layer secrecy approach based on artificial noise, under the constraint of constant envelope beamforming. For the case of one legitimate receiver, they analytically show that the feasibility of the beamforming optimization involved increases with the number of antennas and that the performance of the phase-only beamforming approaches that of the unconstrained beamforming approach.

The second paper by Tourki et. al. studies a collaborative secrecy approach where a secondary link is allowed to share the spectrum of a legitimate link, provided that it helps improve the legitimate link's secrecy against a passive eavesdropper. It derives secrecy outage probability expressions, and shows that these are sufficient for collaboration, while satisfying mutual outage probability conditions is a sufficient condition for both links to gain from the collaboration.

In the third paper, Li et. al. consider a full-duplex twoway relay transmission in the presence of an eavesdropper. They present an artificial-noise approach based on rank-two beamforming to maximize the sum secrecy rate. They adopt a semidefinite relaxation approach and a further low-complexity min-max approach which shows satisfactory performance.

In the fourth paper, Wang et al. provide a fresh view on interference due to densification in massive MIMO aided heterogeneous cloud radio access networks (C-RANs). They derive the area ergodic secrecy rate, secrecy outage probability, and energy efficiency of heterogeneous C-RANs.

The fifth article by Kang et al. proposes a jamming-aided hierarchical cooperation scheme that aims to achieve the optimal scaling law of secrecy capacity in ad hoc networks. The proposed scheme can achieve the maximum throughput scaling with perfect secrecy given a considerable number of eavesdroppers, which is the same as the maximum throughput scaling achieved in the absence of eavesdroppers.

In the sixth article, He et al. study regularized channel inversion (RCI) based precoding schemes for a downlink network where the users simultaneously receive confidential messages and harvest energy. The performance of RCI for power splitting, time switching, and hybrid receiver architectures is analyzed and optimized based on a large system analysis.

The seventh article by Cumanan et. al. proposes a secrecy rate optimization framework for multicasting secrecy networks with cooperative jamming. The optimal strategies for the legitimate transmitter and the jammer are derived by using game theoretic tools.

Li et. al. investigate the maximum achievable secrecy degrees of freedom (SDoF) for the MIMO two-user wiretap interference channel. The maximum achievable SDoF region is derived in closed-form and an achievability scheme that enables cooperation between the two transmitters is proposed.
Zeng et. al. propose a proactive eavesdropping scheme that incorporates a spoofing full-duplex relay to enhance wireless information surveillance. The power splitting ratios and the transmit precoding matrix at the legitimate monitor have been jointly optimized to maximize the achievable eavesdropping rate.

In the tenth article, Zappone et. al. study the energyefficient resource allocation problem in multi-antenna wiretap channels, where the technique of artificial noise is applied for secrecy rate enhancement. For different types of channel state information at the transmitter, the new performance metric termed secrecy energy efficiency is introduced and maximized based on fractional programming and convex optimization techniques.

The eleventh article by Kalantari et. al. studies the precoder design for directional modulation for multi-user MIMO communication systems in the presence of a multi-antenna eavesdropper. It is shown that the proposed symbol level precoding design requires no channel knowledge of the eavesdropper, and yet can enhance the security performance by maximizing the decoding error rate of the eavesdropper.

The last article of this category by Fan et al. studies the problem of opportunistic relay selection in cooperative networks with co-channel interference and wiretapping. Three selection schemes corresponding to different complexities and channel state information is analyzed in terms of secrecy outage probability.

The next three articles deal with the exploitation of interference to enhance the wireless information and power transfer performance of a network. Wang et al. consider a multi-pair two-way relay network where single-antenna users receive information and harvest energy from a multi-antenna relay in the downlink and use the harvested energy for information transmission in the uplink. Minimizing the consumed energy while guaranteeing the quality of service of the users leads to an intricate optimization problem for the relay beamforming vectors, the transmit powers, and the power splitting factor of the energy harvester.

In the fourteenth article, Biswas et al. propose to support outdoor transmission in the mmWave band by wirelessly powered relays in order to mitigate signal blockage. Using tools from stochastic geometry, the performance of the resulting relay-aided mmWave networks is studied in terms of the harvested energy and coverage.

In the fifteenth article, $\mathrm{Bi}$ and Chen study cooperative jamming to improve the PHY layer security performance in wireless communication. Different from the existing work, this paper considers that the friendly jammer has no embedded power supply but is instead powered by harvesting radio frequency energy from the source. The paper proposes a new cooperative jamming protocol termed accumulate-andjam, based on which the dynamic operation of the jammer is optimized to minimize the secrecy communication outage probability.

In the last article of this special issue, Dong et al. develop a modulation division transmission scheme such that each receiver can efficiently detect their desired signals from the superposition of mutually interfering co-channel signals. This 
is achieved by exploring the geometric structure of the sum of pulse amplitude modulation and rectangular quadrature amplitude modulation constellations in the multiple-input singleoutput Gaussian broadcast channel.

Our guest editorial team would like to thank all the authors and reviewers for their contribution to this special issue.

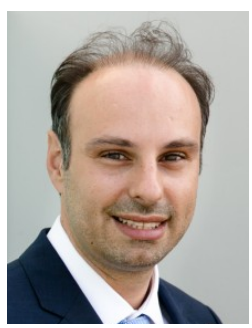

Ioannis Krikidis (S'03-M'07-SM'12) received the diploma in Computer Engineering from the Computer Engineering and Informatics Department (CEID) of the University of Patras, Greece, in 2000, and the M.Sc and Ph.D degrees from Ecole Nationale Superieure des Telecommunications (ENST), Paris, France, in 2001 and 2005, respectively, al in electrical engineering. From 2006 to 2007 he worked, as a Post-Doctoral researcher, with ENST, Paris, France, and from 2007 to 2010 he was a Research Fellow in the School of Engineering and Electronics at the University of Edinburgh, Edinburgh, UK. He is currently an Assistant Professor at the Department of Electrical and Computer Engineering, University of Cyprus, Nicosia, Cyprus. His current research interests include information theory, wireless communications, wireless powered communications, cooperative communications, cognitive radio and secrecy communications.

Dr. Krikidis serves as an Associate Editor for the IEEE Transactions on Communications, IEEE Transactions on Vehicular Technology, and IEEE Wireless Communications Letters. He was the Technical Program Co-Chair for the IEEE International Symposium on Signal Processing and Information Technology 2013. He has published over 150 papers in scientific journals and international conferences and his work is well recognized with more than 2700 citations. He received an IEEE Communications Letters and IEEE Wireless Communications Letters exemplary reviewer certificate in 2012. He was the recipient of the Research Award Young Researcher from the Research Promotion Foundation, Cyprus, in 2013.

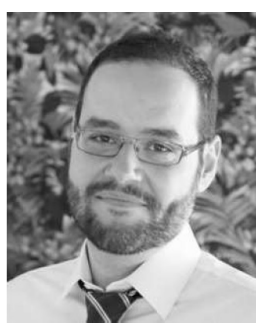

Christos Masouros (M'06, SM'14), is currently a Senior Lecturer in the Dept. of Electrical \& Electronic Eng., University College London. He received his Diploma in Electrical \& Computer Engineering from the University of Patras, Greece, in 2004, MSc by research and $\mathrm{PhD}$ in Electrical \& Electronic Engineering from the University of Manchester, UK in 2006 and 2009 respectively. He has previously held a Research Associate position in University of Manchester, UK and a Research Fellow position in Queen's University Belfast, UK.

He holds a Royal Academy of Engineering Research Fellowship 20112016 and is the principal investigator of the EPSRC project EP/M014150/1 on large scale antenna systems. His research interests lie in the field of wireless communications and signal processing with particular focus on Green Communications, Large Scale Antenna Systems, Cognitive Radio, interference mitigation techniques for MIMO and multicarrier communications. He was the recipient of the Best Paper Award in the IEEE GlobeCom conference 2015, and has been recognised as an Exemplary Reviewer for the IEEE Transactions on Communications. He is an Associate Editor for IEEE Communications Letters.

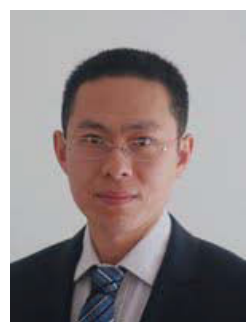

Gan Zheng (S'05, M'09, SM'12) received the BEng and the MEng from Tianjin University, Tianjin, China, in 2002 and 2004, respectively, both in Electronic and Information Engineering, and the $\mathrm{PhD}$ degree in Electrical and Electronic Engineering from The University of Hong Kong in 2008. He is currently a Senior Lecturer in Signal Processing and Networks Research Group in the Wolfson School of Mechanical, Electrical and Manufacturing Engineering, Loughborough University, UK. He had been on various research and visiting positions with University College London, KTH Royal Institute of Technology and University of Luxembourg, and was with University of Essex as a Lecturer in Communications. His research interests include MIMO precoding, cooperative communications, cognitive radio, physical-layer security, full-duplex radio and energy harvesting. Dr. Zheng is a Senior Member of IEEE. He is the first recipient for the 2013 IEEE Signal Processing Letters Best Paper Award, and he also received 2015 GLOBECOM Best Paper Award. He currently serves as an Associate Editor for IEEE Communications Letters.



Rui Zhang (S'00-M'07-SM'15) received the B.Eng. (First-Class Hons.) and M.Eng. degrees from the National University of Singapore in 2000 and 2001, respectively, and the Ph.D. degree from the Stanford University, Stanford, CA USA, in 2007, all in electrical engineering. From 2007 to 2010, he worked at the Institute for Infocomm Research, A-STAR, Singapore, where he now holds a Senior Research Scientist joint appointment. Since 2010, he has joined the Department of Electrical and Computer Engineering of the National University of Singapore as an Assistant Professor. His current research interests include energyefficient and energy-harvesting-enabled wireless communications, wireless information and power transfer, multiuser MIMO, cognitive radio, smart girds, and optimization methods.

Dr. Zhang has published over 200 papers. He was the co-recipient of the Best Paper Award from the IEEE PIMRC in 2005, and the IEEE Marconi Prize Paper Award in Wireless Communications in 2015. He was the recipient of the 6th IEEE Communications Society Asia-Pacific Best Young Researcher Award in 2011, the Young Investigator Award and the Young Researcher Award of the National University of Singapore in 2011 and 2015, respectively. $\mathrm{He}$ has served for over 30 IEEE conferences as TPC members and Organizing Committee members. He is now an elected member of IEEE Signal Processing Society SPCOM and SAM Technical Committees, and serves as the Vice Chair of the IEEE ComSoc Asia-Pacific Board Technical Affairs Committee. $\mathrm{He}$ is an editor for the IEEE Transactions on Wireless Communications, the IEEE Transactions on Signal Processing, and the IEEE Journal on Selected Areas in Communications (Green Communications and Networking Series). 


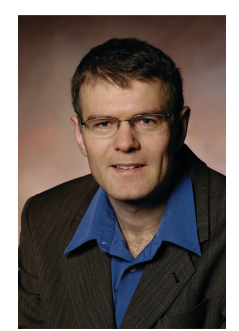

Robert Schober (S'98, M'01, SM'08, F'10) was born in Neuendettelsau, Germany, in 1971. He received the Diplom (Univ.) and the Ph.D. degrees in electrical engineering from Friedrich-AlexanderUniversity Erlangen-Nurnberg (FAU), Germany, in 1997 and 2000, respectively. Since May 2002 he has been with the University of British Columbia (UBC), Vancouver, Canada, where he is now a Full Professor. Since January 2012 he is an Alexander von Humboldt Professor and the Chair for Digital Communication at FAU. His research interests fall into the broad areas of Communication Theory, Wireless Communications, and Statistical Signal Processing.

Dr. Schober received several awards for his work including the 2002 Heinz Maier-Leibnitz Award of the German Science Foundation (DFG), the 2004 Innovations Award of the Vodafone Foundation for Research in Mobile Communications, the 2006 UBC Killam Research Prize, the 2007 Wilhelm Friedrich Bessel Research Award of the Alexander von Humboldt Foundation, the 2008 Charles McDowell Award for Excellence in Research from UBC, a 2011 Alexander von Humboldt Professorship, and a 2012 NSERC E.W.R Steacie Fellowship. In addition, he has received several best paper awards for his research. Dr. Schober is a Fellow of the Canadian Academy of Engineering and a Fellow of the Engineering Institute of Canada. From 2012 to 2015, he served as Editor-in-Chief of the IEEE Transactions on Communications and since 2014, he is the Chair of the Steering Committee of the IEEE Transactions on Molecular, Biological and Multiscale Communication. Furthermore, he is a Member at Large of the Board of Governors of the IEEE Communications Society. 